\title{
Morphological types of hepatocellular hyalin in Indian childhood cirrhosis: an ultrastructural study
}

\author{
N. C. NAYAK AND S. ROY \\ From the Department of Pathology, All India Institute of Medical Sciences, New Delhi, India
}

SUMMARY Morphological variants of intrahepatocytic hyalin in Indian childhood cirrhosis have been analysed by electron microscopy. This structure, morphologically identical with Mallory's alcoholic hyalin, is encountered in three different forms. The most common one is composed of randomly orientated fibrils. The next common type is composed of smudgy homogeneous or finely granular material, while the least common type consists of parallel fibrils with gentle curves giving a 'finger-print' appearance. Fragmented endoplasmic reticulum and ribosomes were frequently associated with hyalin suggesting that these organelles contribute to their formation.

In our earlier studies we drew attention to the frequent occurrence of hyalin in the liver cells in Indian childhood cirrhosis (ICC) and have emphasised its significance in the diagnosis and prognosis of this disease (Nayak et al., 1969, 1972). Histochemical and electron microscopic investigations have demonstrated close similarity between this hyalin and Mallory's hyalin of alcoholic liver disease (Nayak et al., 1969; Roy et al., 1971). The ultrastructure of the latter has been described in several recent reports (Flax and Tisdale, 1964; Biava, 1964; Smuckler, 1968; Yokoo et al., 1972; Wiggers et al., 1973). Yokoo et al. (1972) and others (Wiggers et al., 1973) have demonstrated that alcoholic hyalin exists in different morphological forms. We examined the ultrastructural detail of hyalin in ICC to see if these or other morphological variants are also encountered in this disease.

\section{Methods}

Biopsy specimens of liver from nine cases of Indian childhood cirrhosis were processed for light as well as electron microscopy. In all cases the diagnosis was confirmed and intracytoplasmic hyalin was identified by light microscopic examination. The hyalin was seen randomly distributed without any preferential localization in zones. For electron microscopic examination the tissue was processed according to the methods described earlier (Roy et al., 1971). Elec-

Received for publication 28 June 1976 tron-micrographs of several cytoplasmic hyalin bodies were obtained for morphological analysis.

\section{Results}

The hyalin material generally appeared as irregular masses in the cytoplasm, with varying degrees of electron density in different areas. None showed any membrane around them. They were often spread out over sizeable areas but were occasionally very small and spotty. Close examination revealed three different morphological forms (Fig. 1). The most frequent one occurred as irregular masses of randomly orientated interwoven fibrils, admixed with electron dense granules (Figs. 1 and 2). The outline of these was usually not clearly demarcated from their surroundings, where degenerating cell organelles, especially the rough endoplasmic reticulum, as well as free ribosomes were frequently observed (Fig. 3). Occasionally, the organelles were seen embedded within the hyalin mass. The fibrils comprising this form of hyalin measured approximately $20 \mathrm{~nm}$ in diameter. At a higher magnification some of these fibrils appeared to be tubular, particularly in transverse section (Fig. 2). The granules were of almost the same diameter as the fibrils but many of them appeared more dense.

The next common form of hyalin appeared as smudgy, homogeneous or finely granular electron dense material with no discernible fibrillary structure (Figs, 1, 3-6). They also had an irregular outline and showed degenerating organelles at the periphery 


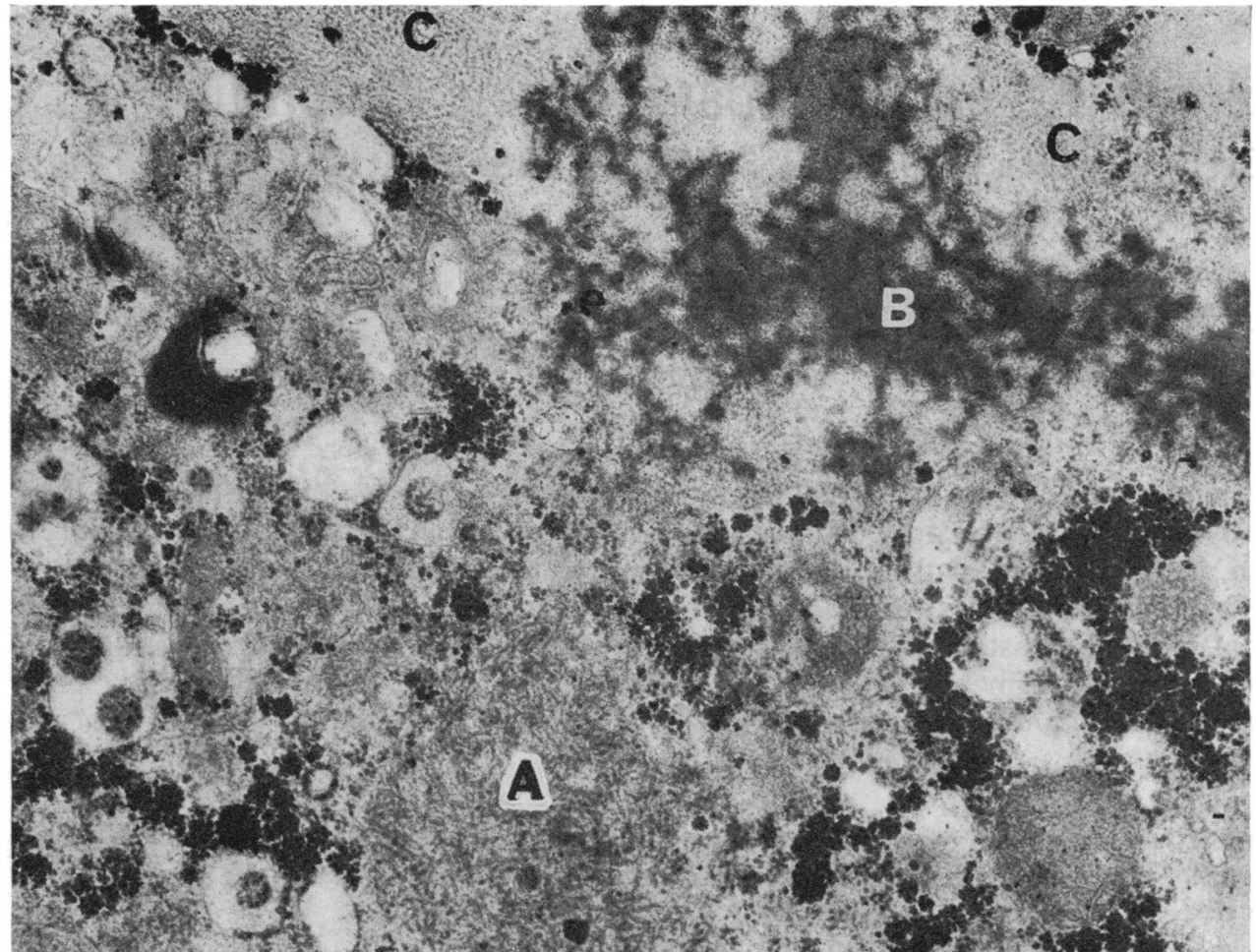

Fig 1

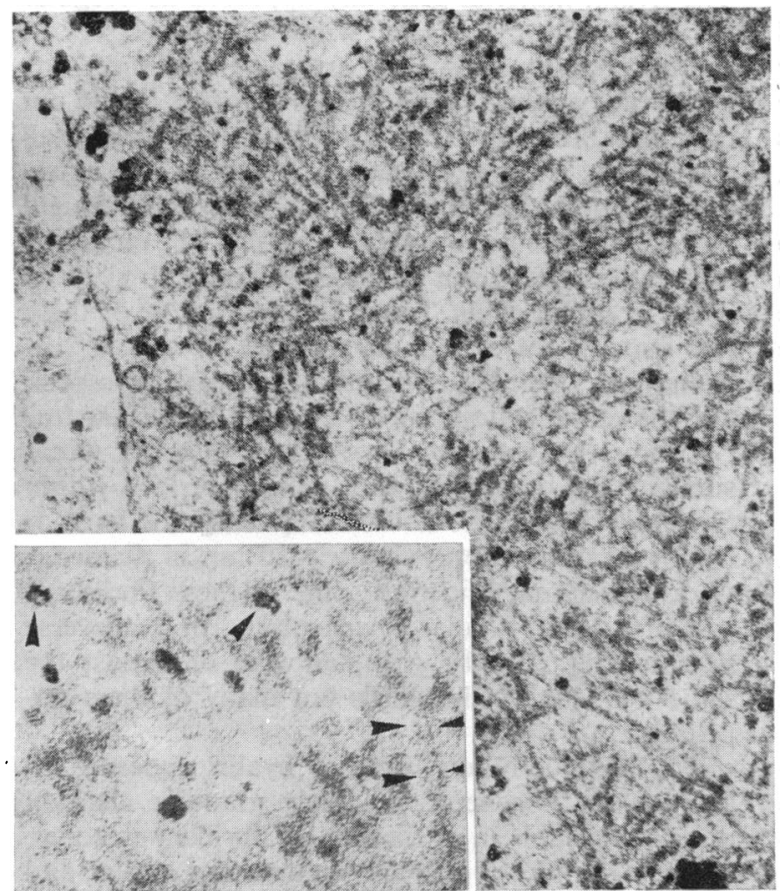

Fig 2
Fig. 1 Electron micrograph of a hepatocyte showing three forms of hyalin $(A, B$, and $C)$. None of them is membrane bound. The hyalin in $A$ and $C$ is composed of fibrils, while that in $B$ is made up of smudgy homogeneous electron dense material. The fibrils in $A$ are randomly orientated but in $C$ they are parallel with gentle curves giving a 'finger-print' appearance. $\times 25000$.

Fig. 2 Higher magnification of hyalin with randomly orientated fibrils and granules with variable electron density. $\times 51000$. Inset; some fibrils show tubular configuration (arrow heads). $\times 114000$. 

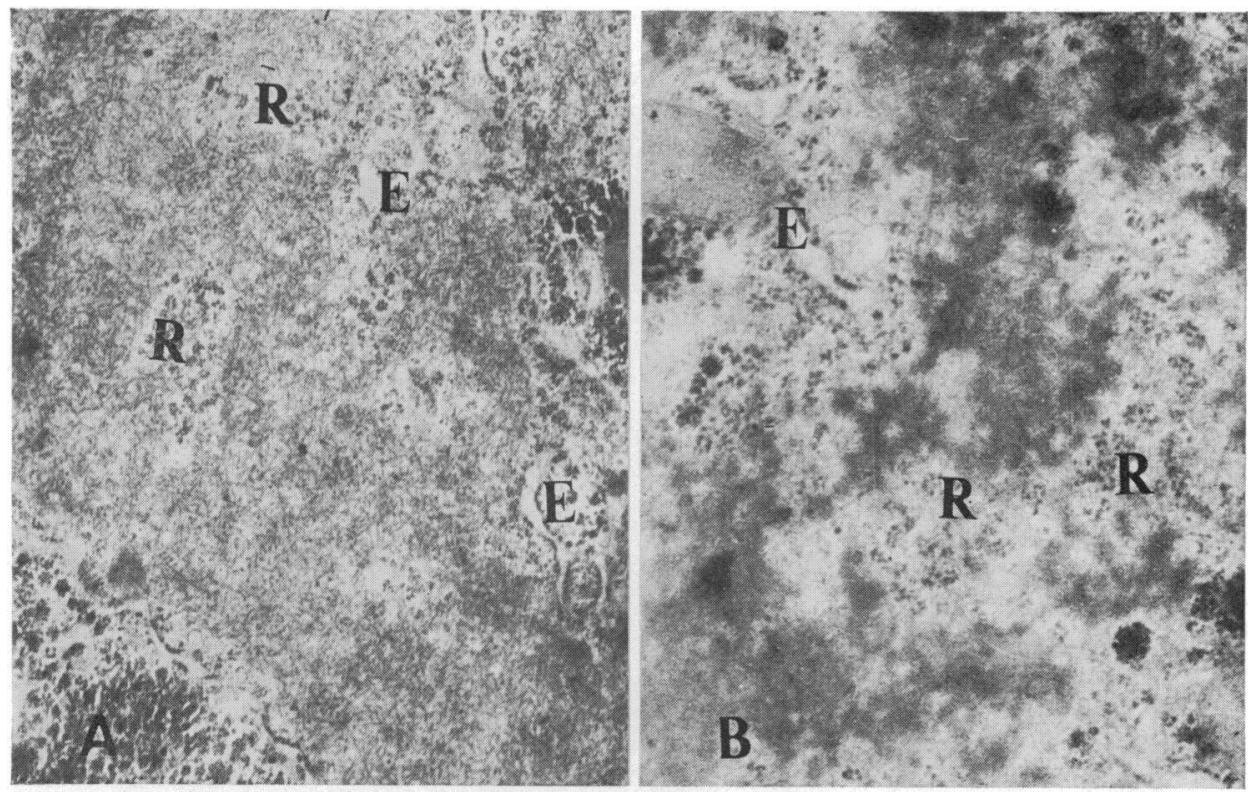

Fig. 3 A. Hyalin with randomly orientated fibrils. B. Hyalin composed of smudgy homogeneous material. Note the presence of remnants of rough endoplasmic reticulum $(E)$ and free ribosomes $(R)$ at the periphery and inside the hyalin mass in both types. $A ; \times 16000 . B ; \times 17000$.

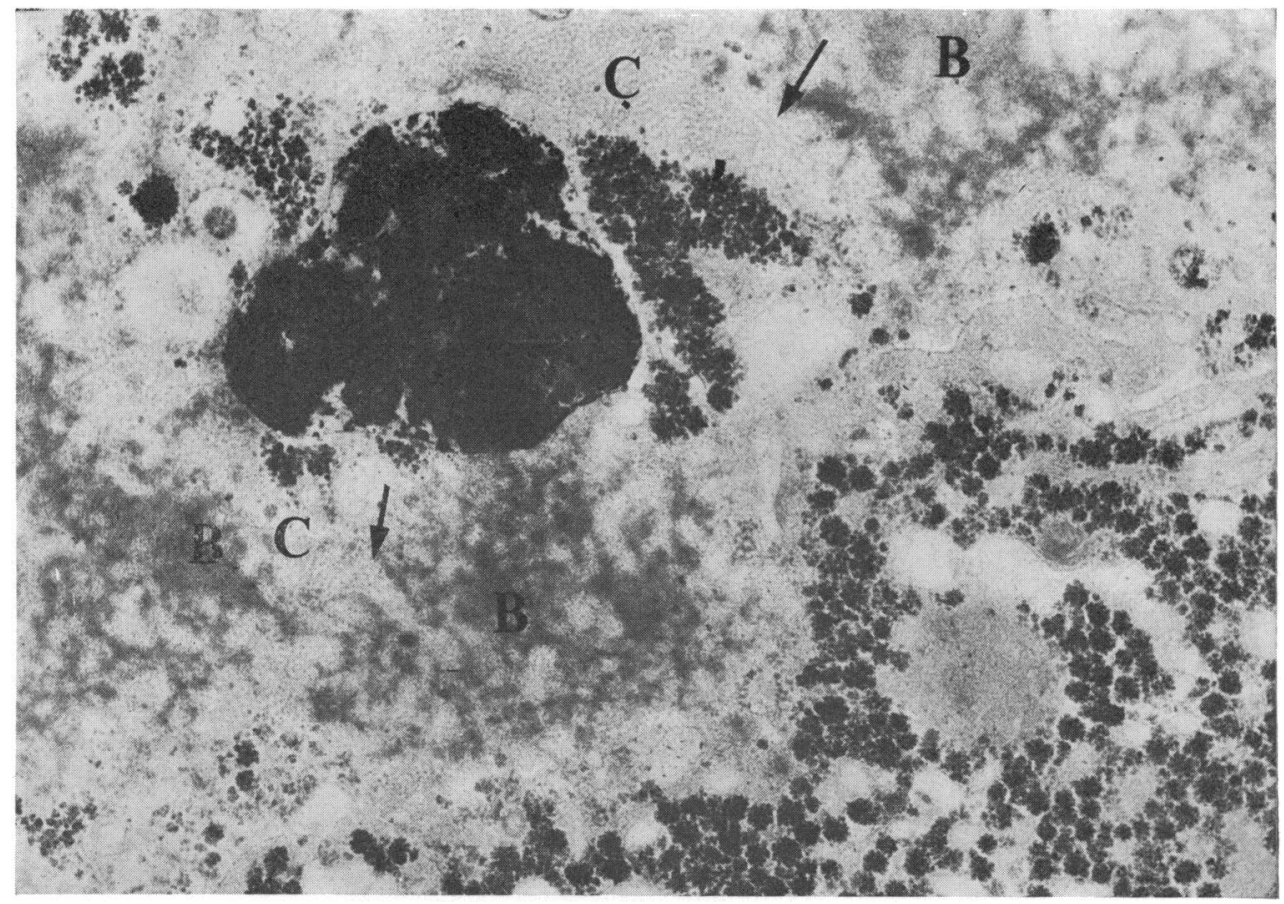

Fig. 4 Two types of hyalin composed of homogeneous material $(B)$ and of fibrils with parallel arrangements $(C)$. The latter is in close approximation to the former in some areas (arrows). $\times 25000$. 


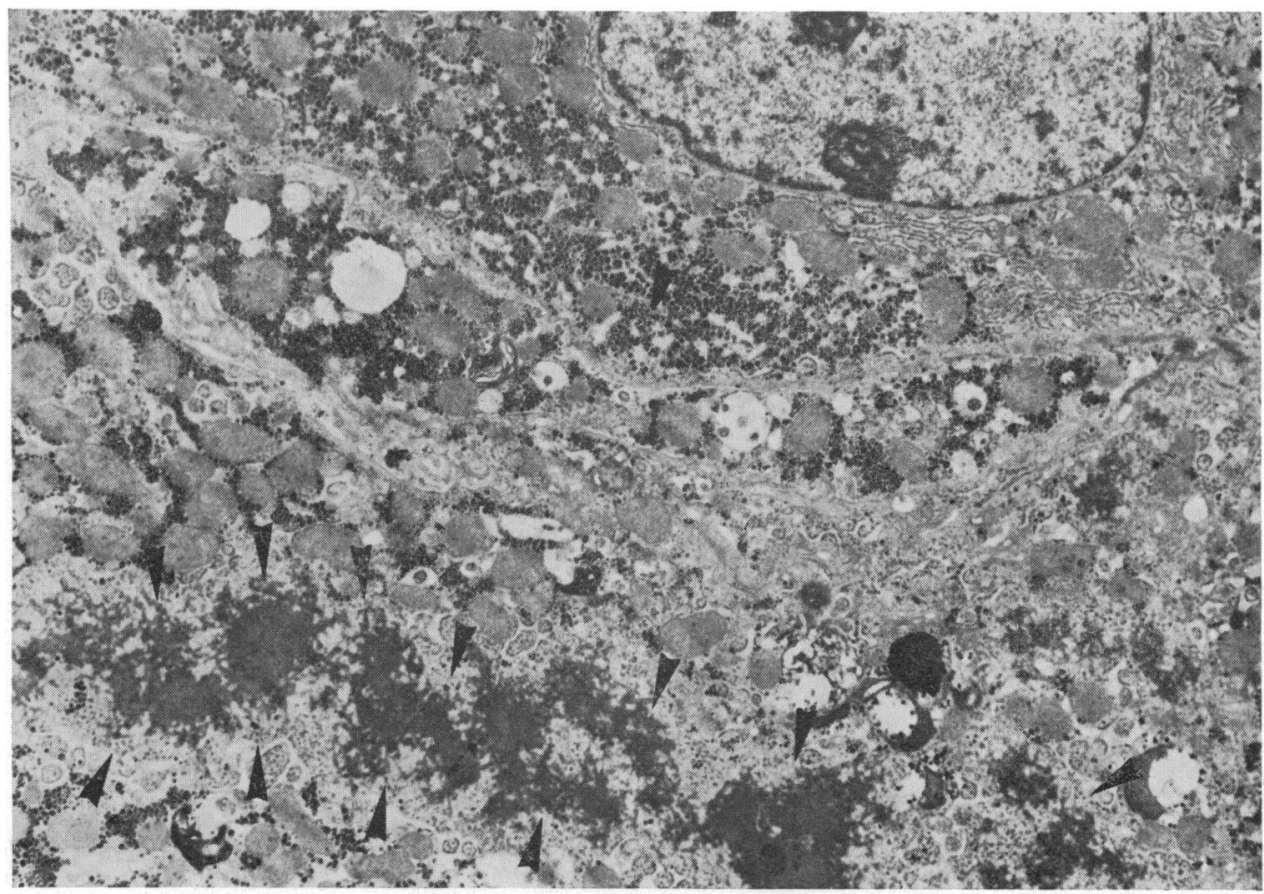

Fig. 5 Hepatocyte with hyalin containing homogeneous material that spreads over a large area in the cytoplasm (within arrow head). Note degenerating organelles including rough endoplasmic reticulum and ribosomes at the periphery. $\times 6600$.

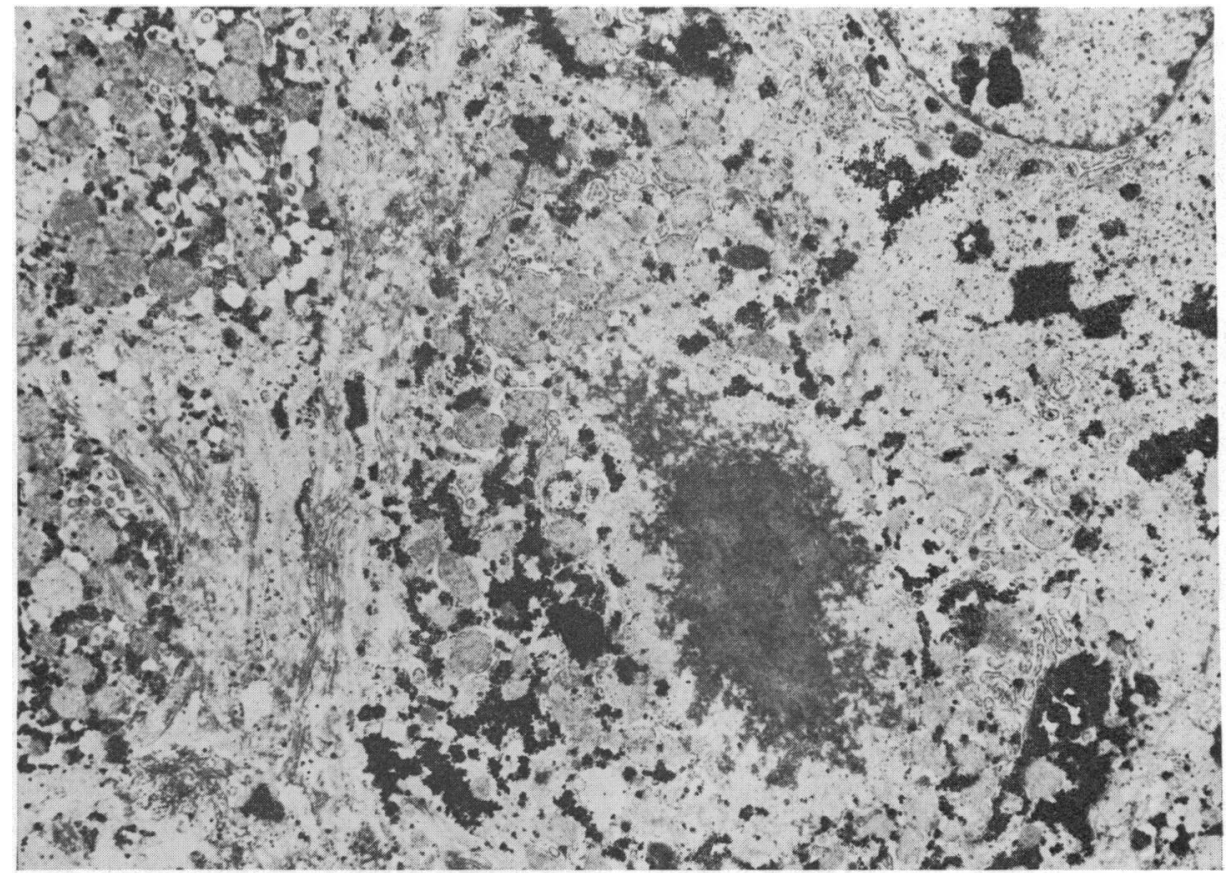

Fig. 6 Hepatocyte containing a small hyalin body composed of homogeneous material with degenerating organelles at the periphery. $\times 6000$. 
(Fig. 5). Occasionally, very small accumulations of hyalin consisted almost entirely of this type (Fig. 6). In a few areas somewhat swollen and indistinct, randomly orientated fibrils of the type described earlier could be seen at the peripheral part. In some hepatocytes these collections extended irregularly over a large area of the cytoplasm (Fig. 5) interspersed with small pockets containing organelles which appeared to be either normal or degenerated.

The third and the least common type of hyalin appeared as closely packed bundles of fine fibrils arranged in parallel arrays (Figs. 1 and 4). These collections were usually smaller than those containing the other two types of hyalin and their margins were often more regular. The fibrils were about the same width as in the first type of hyalin measuring on average $18 \mathrm{~nm}$ in diameter. They frequently presented a beaded appearance and showed parallel arrangements with gentle curves, imparting a 'finger-print' pattern to the hyalin. The fibrils of this type of hyalin were less electron dense than those in the other types described above.

It should be pointed out that, although any one of the three types of hyalin could alone be present in a hepatocyte, stimultaneous occurrence of more than one type in a cell was not unusual.

\section{Discussion}

Several electron microscopic studies have established that alcoholic hyalin is an intracytoplasmic fibrillar deposit, not bounded by a limiting membrane (Flax and Tisdale, 1964; Biava, 1964; Smuckler, 1968; Isrei and Gottlieb, 1971; Yokoo et al., 1972). Hyalin with identical fibrillar structure has also been demonstrated in certain non-alcoholic liver diseases (Gerber et al., 1973). A Mallory body of this type has also lately been described in griseofulvininduced liver injury in mice (Denk et al., 1975). Yokoo et al. (1972) have described three variants of alcoholic hyalin-namely, types 1,2 , and 3 . Of these only types 1 and 2 are fibrillar, while type 3 is homogeneous or granular. Type 1 differs from type 2 mainly in the mode of arrangement and density of the fibrils. In type 1, the fibrils are aligned in parallel arrays, while in type 2 they are randomly oriented and are denser than those in type 1. Identical types of hyalin have also been observed by Wiggers et al. (1973) in alcoholic liver disease.

In the present study, too, the three forms of hyalin could be detected in the hepatocytes from cases of ICC. In the study of Yokoo et al. (1972) hyalin was present in hepatocytes, bile ductular cells, and in benign and malignant hepatomas. However, in ICC we have not yet observed hyalin in cells other than the hepatocytes.
In our material, hyalin bodies composed of interwoven bundles of fibrils and irregular masses of homogeneous material (types 2 and 3 of Yokoo et al.) were seen almost equally frequently, while the 'finger-print' type (type 1 of Yokoo) was only occasionally found. In alcoholic liver disease, in addition to the presence of hyalin, bundles of thin filaments of a much smaller diameter than the fibrils of hyalin frequently occurred. In ICC we found no such association.

Yokoo et al. (1972) considered that all the three forms of hyalin seen in alcoholic liver are closely related and suggested that the two fibrillar forms of hyalin result from two different types of orientation of the fibrils, and in the third form the fibrillar character is lost. This latter form was thus interpreted as being a more advanced stage of hyalin formation. However, in our study, the homogeneous form (type 3 ) could be frequently seen in very small areas with no obvious fibrils in the vicinity. Therefore, it seems to us that this type can form de novo and need not necessarily evolve from the fibrillar form. The other two forms may, however, represent the two modes of orientation of basically the same fibrillar material, since the fibrils in both types are of almost the same thickness. It is not clear why the hyalin with fibrils showing parallel orientation is so rare even in cases where almost every hepatocyte contains the other forms of hyalin. Some of the fibrils in our material presented a tubular appearance. This has also been emphasised in similar hyalin of livers in chronic alcoholics (Biava, 1964; Wiggers et al., 1973), studied in situ as well as after centrifugation.

In spite of a large number of studies on alcoholic hyalin, the way in which it is formed has not been established. In our earlier studies we suggested the possibility that it was formed from endoplasmic reticulum (Nayak et al., 1969; Roy et al., 1971). In the present study, too, degenerating and fragmented segments of rough endoplasmic reticulum could be frequently seen around or within hyalin masses, especially of the fibrillar form (type 2 of Yokoo). Moreover, electron dense granules resembling ribosomes were frequently present in the fibrillar form of hyalin masses. Therefore, the possibility of the contribution of endoplasmic reticulum cannot be excluded (Biava, 1964; Roy et al., 1971; Nayak et al., 1969). French and Davies (1974) have suggested that ribosomes synthesise the protein of alcoholic hyalin, though the morphological association of the two may later become obscured by the enlarging hyalin body. It has been suggested that the fibrils in hyalin are synthesised by the liver cell, representing a contractile system of the cells (Yokoo et al., 1972; Wiggers et al., 1973). However, more recent 
chemical studies have shown that the fibrils are not related to actin and they may represent the intermediate filaments of the liver cells (French and Davies, 1974). The mechanism of formation of the homogeneous form of hyalin is not clear. This probably represents another form of degenerative change in the cytoplasm.

The authors are grateful to Mr Achhar Singh for expert technical help.

\section{References}

Biava, C. (1964). Mallory alcoholic hyalin: a heretofore unique lesion of hepatocellular ergastoplasm. Laboratory Investigation, 13, 301-320.

Denk, H., Gschnait, F., and Wolff, K. (1975). Hepatocellular hyalin (Mallory bodies) in long term griseofulvin-treated mice: a new experimental model for the study of hyalin formation. Laboratory Investigation, 32, 773-776.

Flax, M. H., and Tisdale, W. A. (1964). An electron microscopic study of alcoholic hyaline. American Journal of Pathology, 44, 441-453.

French, S. W., and Davies, P. L. (1974). The Mallory body in the pathogenesis of alcoholic liver disease. In Biochemical, Epidemiological and Clinical Aspects of the Etiology of Alcoholic Liver Pathology. Edited by Y. Israel. (In press). Quoted by E. Rubin and C. S. Lieber. In Clinics in Gastroenterology, 1975.
Gerber, M. A., Orr, W., Denk, H., Schaffner, F., and Popper, H. (1973). Hepatocellular hyalin in cholestasis and cirrhosis: its diagnostic significance. Gastroenterology, 64, 89-98.

Iseri, O. A., and Gottlieb, L. S. (1971). Alcoholic hyalin and megamitochondria as separate and distinct entities in liver disease associated with alcoholism. Gastroenterology, 60, 1027-1035.

Nayak, N. C., Sagreiya, K., and Ramalingaswami, V. (1969). Indian childhood cirrhosis: the nature and significance of cytoplasmic hyaline of hepatocytes. Archives of Pathology, 88, 631-637.

Nayak, N. C., Visalakshi, S., Singh, M., Chawla, V., Chandra, R. K., and Ramalingaswami, V. (1972). Indian childhood cirrhosis: a re-evaluation of its pathomorphologic features and their significance in the light of clinical data and natural history of the disease. Indian Journal of Medical Research, 60, 246-259.

Roy, S., Ramalingaswami, V., and Nayak, N. C. (1971). An ultrastructural study of the liver in Indian childhood cirrhosis with particular reference to the structure of cytoplasmic hyaline. Gut, 12, 693-701.

Smuckler, E. A. (1968). The ultrastructure of human alcoholic hyalin. American Journal of Clinical Pathology, 49, 790-797.

Wiggers, K. D., French, S. W., French, B. A., and Carr, B. N. (1973). The ultrastructure of Mallory body filaments. Laboratory Investigation, 29, 652-658.

Yokoo, H., Minick, O. T., Batti, F., and Kent, G. (1972). Morphologic variants of alcoholic hyalin. American Journal of Pathology, 69, 25-40. 